\title{
O USO DO ORGANIZATIONAL KNOWLEDGE ASSESSMENT (OKA) PARA ANÁLISE DA GESTÃO DO CONHECIMENTO EM UMA ORGANIZAÇÃO PÚBLICA
}

Data de submissão: 28/05/2014 Aceite: 02/08/2016

Oscar Pedro Neves Júnior ${ }^{1}$ Sidnei Vieira Marinho²

\section{RESUMO}

A Gestão do Conhecimento nas organizações está se tornando cada vez mais importante para sustentar a vantagem competitiva e a eficiência de seus processos. Assim, cada vez mais é necessário identificar o nível da Gestão do Conhecimento (GC) nas organizações. Para que isto ocorra, é preciso que as organizações públicas façam um diagnóstico nas práticas de GC, como forma de verificar e melhorar a qualidade de suas informações. O artigo aborda o tema na Administração Pública, Secretaria de Defesa Civil, com o objetivo de quantificar as práticas da GC através do método OKA. O método objetiva identificar a situação da GC nas organizações através da aplicação de um questionário com 199 questões, baseandose em métricas elaboradas para medir cada uma das dimensões estabelecidas. Os resultados da pesquisa mostraram que é necessário que a organização promova a implementação e o compartilhamento das práticas de GC, instigando o fluxo de informações, facilitando a comunicação, o alinhamento das estratégias e estabelecendo objetivos comuns com todos os funcionários.

PALAVRAS-CHAVE: Gestão do Conhecimento, Método OKA, Defesa Civil.

\footnotetext{
1 Possui graduação em Ciência da Computação pela Universidade do Sul de Santa Catarina, mestrado em Administração pela Universidade do Vale do Itajaí. Capivari de Baixo - SC - Brasil. E-mail: oscarnjr@gmail.com

2 Possui graduação em Engenharia de Produção Mecânica pela Universidade Federal de Santa Catarina, mestrado em Engenharia de Produção pela Universidade Federal de Santa Catarina e doutorado em Engenharia de Produção pela Universidade Federal de Santa Catarina. São José - SC. E-mail: sidnei@univali.br
} 


\section{INTRODUÇÃO}

Diante de um cenário de avanços tecnológicos, principalmente na área da informação e comunicação, elaborar políticas que promovam a qualidade dos serviços de atendimentos emergenciais, essencialmente os de segurança da sociedade, é fator primordial para a melhoria da qualidade de vida do ser humano. $O$ atual modelo de crescimento econômico, os riscos e impactos das mudanças climáticas e a preeminente necessidade de focar em uma economia sustentável trazem desafios na criação de valores dentro das empresas (PINSKY; KRUGLIANSKAS, 2013).

Davenport e Völpel (2001) afirmam que a criação do valor se inicia pelo êxito da Gestão do Conhecimento, onde as organizações precisam criar conjuntos de funções e qualificações para desempenhar o trabalho de aprender, distribuir e aplicar o conhecimento, apresentando diferentes tipos de projetos, suportes as estruturas organizacionais, tecnologias efetivas que permitam a criação de valor e apontar direções futuras. A Gestão do Conhecimento não é uma disciplina única, é um campo integrado que se baseia em diversas áreas que permitem buscar iniciativas nos diversos domínios e níveis da hierarquia organizacional (ANSUATTIGUI; PITHON, 2011).

A Gestão do Conhecimento permite às organizações públicas mensurar, com mais segurança, a sua eficiência, tomar decisões acertadas com relação a melhor estratégia a ser adotada junto aos seus clientes e serviços, saber identificar as fontes de informações, saber administrar dados e informações e saber gerenciar seus conhecimentos (SANTOS, 2012).

Batista et al. (2005), em pesquisa realizada sobre Gestão do Conhecimento na Administração Pública, escreveu que priorizar a Gestão do Conhecimento é o grande desafio para a maioria dos órgãos da Administração Direta. Além de áreas administrativas e de planejamento, terão de integrar a Gestão do Conhecimento com as atividades do gerenciamento da rotina do trabalho.

Neste sentido, as organizações públicas vêm passando por inúmeras transformações, necessitando de uma melhor compreensão cognitiva entre homem e tecnologia para interagir neste cenário de constantes mudanças e desenvolvendo competências para conhecer, planejar e intervir nos desastres naturais e humanos atendidos pela Defesa Civil de Santa Catarina. Uma das grandes dificuldades dos gestores da Defesa Civil é o uso das informações no trabalho estratégico para emprego de seus recursos. Isto ocorre principalmente na grande quantidade de setores e colaboradores envolvidos, no volume de informações que cada uma detém e na ausência de práticas de GC (CALHEIROS; CASTRO; DANTAS, 2009).

Dessa forma, com a implantação das práticas de Gestão do Conhecimento na Secretaria de Estado de Defesa Civil é possível que os gestores aumentem a qualidade decisória com o uso das informações das entidades envolvidas nos municípios catarinenses. Rossetti e Morales (2012) afirmam que os gestores devem focar no contexto da estratégia organizacional, direcionando-a na obtenção de melhoria do desempenho organizacional, a partir de uma visão integrada de GC, tanto nos planos das estruturas funcionais e de comando, quanto relativo aos processos, pela busca do alinhamento entre práticas de GC e outras áreas voltadas à agregação de valor.

Contudo, os diagnósticos dos fatores críticos nos processos da empresa somente possibilitarão impactar na adoção de práticas e atividades relacionadas a GC, se for objeto cuidadoso no planejamento e execução através de um diagnóstico organizacional do conhecimento, como prática fundamental e que deve, obrigatoriamente, figurar como primeira etapa do planejamento da Gestão do Conhecimento (PEREZ-SOLTEIRO et al., 2009).

O desafio está em desenvolver pessoas com o perfil requerido para este novo tipo de organização. É fundamental repensar os papéis dos gestores públicos e funcionários dessa or- 
ganização, criar novos sistemas de gestão, fazer com que o aprendizado seja parte do dia a dia da organização. Portanto, o sucesso de um programa de Gestão do Conhecimento em uma organização somente ocorrerá se esta fizer um diagnóstico para conhecer estas práticas de gestão (FRESNEDA, et al., 2008).

Nesta perspectiva, emerge a questão norteadora para o presente estudo: Como ocorrem as práticas da Gestão do Conhecimento na Secretaria de Defesa Civil de Santa Catarina? Dessa forma, o presente artigo tem como objetivo central analisar a Gestão do Conhecimento numa organização pública, Secretaria de Defesa Civil de Santa Catarina, através de uma ferramenta de avaliação, no sentido de informar aos gestores como estão às práticas de Gestão do Conhecimento e, a partir dos resultados, facilitar na elaboração de um plano de gerenciamento do conhecimento.

\section{REFERENCIAL TEÓRICO}

Nos estudos da Gestão do Conhecimento, segundo Grotto (2001), um dos grandes desafios é promover o compartilhamento do conhecimento que não é encontrado nos manuais, nos relatórios e nas pesquisas. Por ser um conhecimento de difícil captação, formalização e comunicação, diligenciar seu compartilhamento pode requerer mudança e mobilização de toda a organização.

A interação entre pessoas, tecnologias e processos é o suporte para que uma organização execute novas e complexas tarefas. Ao gestor compete coordenar os diferentes níveis de conhecimento entre os integrantes de um grupo, através do compartilhamento da informação, considerando que é através de pessoas que esta pode ser interpretada e transformada em conhecimento (BHATT, 2001).

Gestão do Conhecimento é um princípio gerencial que permite ampliar informação, conhecimento, experiência e intuição nas organizações a fim de gerar valor. Sua importância decorre do fortalecimento do conhecimento, com base para vantagem competitiva e sustentável nas organizações (FONSECA et al., 2010).

No entanto, Schreiber (2002) descreve conhecimento como o conjunto de dados e informações que as pessoas usam para suas ações, realizando tarefas e gerando mais informações. O conhecimento concerne ao conjunto completo de informações, dados e relações que levam as pessoas à tomada de decisão, à realização de tarefas e à criação de novas informações ou conhecimentos (FIALHO, 2006).

Para Silveira (2011), a Gestão do Conhecimento tem como intuito criar, identificar, integrar, capturar, compartilhar e mensurar as experiências e o conhecimento existente na organização, pela utilização de metodologias e tecnologias específicas. Uma das maneiras de enfrentar as mudanças sucessivas que ocorrem no seu ambiente é originar, obter e aplicar conhecimento de forma contínua. Dessa forma, possibilita criar produtos, processos, apurar qualidade, novos arranjos organizacionais, novas habilidades, novas crenças, novos valores, novos aprendizados, novas atitudes e comportamentos como também novas vantagens competitivas sustentáveis. A finalidade é obter uma perspectiva de alcance de resultados superiores (NONAKA; TAKEUCHI, 1997).

A Gestão do Conhecimento conduz a empresa nos seus processos e gera capacidade criativa implantando práticas que facilitem o alcance dos objetivos de diferenciação competitiva. Por intermédio da Gestão do Conhecimento é possível identificar as deficiências internas e elaborar um planejamento voltado às ações corretivas (CRUZ, 2002; FIALHO et al., 2006; GROTTO, 2001).

Ao verificar o conceito de Boff (2000), onde a Gestão do Conhecimento por vezes é por demais ampla e demonstra a noção de integração dentro de um processo contínuo de geração 
e uso de conhecimento, que pode ser melhor compreendido num ciclo permanente de várias ações. Este conjunto de ações cria na empresa um ambiente de conhecimento que passa a fazer parte da própria empresa. Esse ambiente propicia a criação do conhecimento, representa o processo de geração da riqueza (BUKOWITZ; WILLIANS, 2002).

Através dos diversos conceitos estudados (NONAKA, TAKEUCHI, 1997; BOFF, 2000; BHATT, 2001; FONSECA et al., 2010; SCHREIBER, 2002; SILVEIRA, 2011) entende-se a Gestão do Conhecimento como um processo, cujo propósito é criar riqueza e valor estratégico em busca da vantagem competitiva para a organização, envolvendo tecnologias, aprendizagem organizacional, criatividade, às competências fundamentais para a construção do conhecimento, educação corporativa, novas metodologias e ferramentas, entre outros aspectos.

\subsection{Práticas e Estratégias da Gestão do Conhecimento}

O emprego dos saberes refere-se à capacidade da organização de explorar seus conhecimentos e, com isso, gerar mais aplicações e melhores resultados. Dessa forma, o uso do pensamento ocorre no processo de integração por indivíduos em sua prática diária na organização.

Leocádio (2011) diz que a caracterização dos processos de criação, disseminação e uso do conhecimento permitem definir a abordagem da Gestão do Conhecimento adotada, e serve como ponto de partida para identificar e analisar as práticas do conhecimento. Sendo assim, tal qual o processo de inovação, a Gestão do Conhecimento é a investigação contínua no caminho evolutivo, tendo como pilares os princípios da aplicação das ideias entre pessoas nos diversos setores das empresas (PARK; KIM, 2005).

No entanto, para Coombs e Hull (1998), para que haja interação entre os indivíduos, é necessário compreender as rotinas organizacionais como qualquer ação que possua algumas características, como: são executadas regularmente; baseiam-se em padrões formais ou informais de trabalho; podem ou não possuir um suporte tecnológico; possibilitam a criação, disseminação e uso do conhecimento e facilitam o alcance dos objetivos da organização. Com isso, essas rotinas organizacionais formais ou informais identificam como as organizações promovem a interação entre pessoas, processos e tecnologias na Gestão do Conhecimento.

Já o estudo estratégico relacionado aos processos e infraestruturas da empresa é empregado para adquirir, criar e compartilhar ideias para o planejamento e tomada de decisões, conectando conhecimento estratégico para tática de negócios. O planejamento do conhecimento da empresa descreve a aproximação global que uma organização pretende tomar para alinhar seus recursos de saberes e capacidades intelectuais necessárias reduzindo, assim, o entendimento existente entre o que uma empresa deve saber para executar sua estratégia e o que ela sabe (ZACK; 1999, SCHULTZ, 2011).

Os autores Bierly e Daly (2002) mostram semelhante definição, onde o conjunto de escolhas abordado na criação do conhecimento em uma organização compreende estratégias da Gestão do Conhecimento da empresa, que fornecem as mesmas orientações para criar vantagem competitiva. Ambas as definições levam em conta a conveniência do gerenciamento do conhecimento explícito com o claro objetivo estratégico. Geralmente, a Gestão do Conhecimento é sempre adotada inconscientemente durante a trajetória da empresa. (GARAVELLI; GORGOGLIONE; SCOZZI, 2004).

Os autores Bierly e Chakrabarti (1996) rotularam as empresas de acordo com a forma que gerenciaram conhecimento. Eles concluíram que estratégias do conhecimento mais agressivas caracterizavam instituições mais inovadoras, ocasionando alto desempenho nos negócios. 
Da mesma forma, Zack (1999) propôs duas orientações: conservadoras versus agressivas, na qual as primeiras são empresas conservadoras com foco estratégico na exploração do conhecimento interno, enquanto que as segundas são agressivas e integram estritamente a exploração de conhecimento e a utilização sem ter em conta os limites organizacionais.

Um segundo destaque ocorre ao incluir classificação significativa de exploração versus prospecção por March (1991), onde relaciona a distinção entre conhecimento tácito e explícito (DAVENPORT; VÖLPEL, 2001). Além, dos conceitos de personalização e codificação do conhecimento são facilmente entendidos por acadêmicos e praticantes.

Ao se trabalhar a estratégia, verifica-se a capacidade do poder organizacional, entendido como os recursos disponíveis na organização, os que ela tem de reserva e os que ela pode mobilizar para atingir os objetivos.

\subsection{Metodologia para Diagnóstico da Gestão do Conhecimento}

O Banco Mundial, por intermédio do World Bank Institute (WBI), desenvolveu o método Organizational Knowledge Assessment (OKA), como forma de diagnosticar a situação da GC nas organizações. Esta ferramenta possibilita a coleta de dados sobre os vários aspectos da GC, facilitando a análise destes através do uso de gráficos do tipo radar.

De acordo com Fresneda e Gonçalves (2007), existem três possíveis estratégias de formulação de um Plano de Gestão do Conhecimento (PGC) a partir das informações coletadas, e mais especificamente da análise inicial do diagrama final, que são: a) Analisar os pontos fracos da organização que são representados pelas dimensões do método OKA onde foram obtidas as menores pontuações e representados no diagrama com os pontos para dentro na figura e, a partir desta análise, gerar um plano de ação; b) Analisar os pontos fortes da organização que são representados pelas dimensões do método OKA onde foram obtidas as maiores pontuações e representados no diagrama com os pontos extremos para fora da figura e, a partir desta análise, gerar um plano de ação; c) Analisar os pontos fracos e fortes da organização, conforme itens (a) e (b) acima e, a partir desta análise, gerar um plano de ação que é um composto das duas estratégias anteriores. Em qualquer escolha, deve-se levar em conta sempre a análise do alinhamento entre os objetivos da organização e as dimensões do conhecimento necessárias para a implantação de um plano de GC (FRESNEDA; GONÇALVES, 2007). Vale ressaltar que esse método não tem relação com os modelos clássicos, como da criação do conhecimento de Nonaka e Takeuchi (1995), tratase de um método da avaliação da Gestão do Conhecimento dentro da organização, independente de ocorrer práticas formais ou informais ou do modelo aplicado de Gestão do Conhecimento.

Para Fresneda e Gonçalves (2007), o método de Avaliação da Gestão do Conhecimento Organizacional - OKA é uma ferramenta apropriada para efetuar o diagnóstico nas organizações e, a partir da análise dos dados coletados, é possível estabelecer as estratégias e ações para meIhorar ou elaborar o Plano de GC da instituição.

O autor Wiig (2000) corrobora com essa ideia ao mencionar que o uso do diagrama gerado pelo método OKA demonstra a situação da GC na organização e seu diagnóstico para poder ser utilizado em inúmeros propósitos, como: situação da GC, definição de estratégias, priorização de projetos, bem como na identificação de necessidades e oportunidades específicas no contexto da GC.

De acordo com Fonseca (2006), uma organização deve esforçar-se para quantificar sua capacidade de identificar informação, conhecimento, experiência e intuição por meio das Pessoas, Processos e Sistemas para alcançar seus objetivos e gerar valor. Dessa forma é que se apresenta o método OKA, um instrumento para quantificar onde a Gestão do Conhecimento impacta 
e é dependente, essencialmente em três elementos que compõem uma organização: Pessoas, Processos e Sistemas (figura 1).

Figura 1 - Arquitetura do método OKA

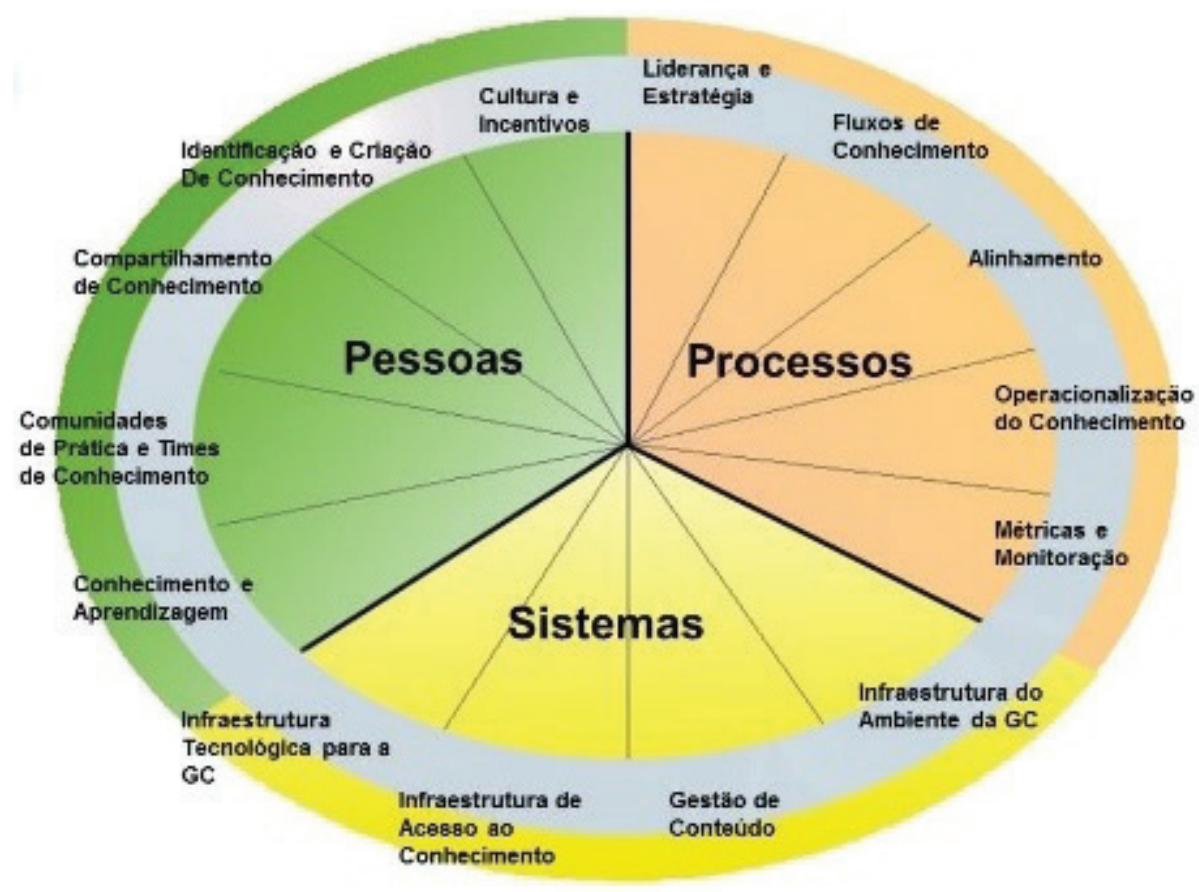

Fonte: Traduzido de Fonseca, 2006

A figura 1, além de apresentar os três elementos básicos: Pessoas, Processos e Sistemas, é composta por uma série de 14 dimensões do conhecimento, distribuídas em 199 questões com pontuações associadas a um valor para cada questão, são essas: Cultura e Incentivos, Identificação e Criação do Conhecimento, Compartilhamento do Conhecimento, Comunidades de Prática e Equipes de Conhecimento, Conhecimento e Aprendizagem, Liderança e Estratégia, Fluxo de Conhecimento, Operacionalização do Conhecimento, Alinhamento, Indicadores e Monitoramento, Infraestrutura Tecnológica da GC, Infraestrutura de Acesso ao Conhecimento, Gestão do Conteúdo e Infraestrutura do Ambiente de GC. Essa série de dimensões do conhecimento é que constituem o elemento-chave do método, que por sua vez é caracterizado por métricas bem definidas, que foram escolhidas como resultados de pesquisas e estudos na área de Gestão do Conhecimento (FONSECA, 2006).

O entendimento de Fresneda e Gonçalves (2007) de que a partir de um diagnóstico inicial elaborado pelo método OKA e da análise dos dados coletados, é possível definir as ações para o desenvolvimento das práticas de GC na organização. A contribuição principal do método OKA é quantificar a capacidade de uma instituição de promover o uso da informação, conhecimento, experiência e intuição por meio de Pessoas, Processos e Sistemas com o objetivo de atingir seus objetivos e gerar valor (BATISTA, 2008).

Sendo assim, no capítulo seguinte, apresenta-se as fases de aplicação do Método de Avaliação do Conhecimento (OKA). 


\section{METODOLOGIA DA PESQUISA}

Para realização deste trabalho foi realizada pesquisa exploratória e bibliográfica. Como instrumento de coleta de dados foi utilizado o método Organizational Knowledge Assesstment (OKA) - Avaliação do conhecimento Organizacional.

Ao final da coleta de dados, o conjunto de respostas obtidas com a aplicação da ferramenta OKA é submetido a uma metodologia de apuração a qual atribui um valor numérico a cada uma das quatorze dimensões do conhecimento avaliadas. A partir destes valores é gerado um gráfico tipo radar (gráfico 1), cuja análise demonstra as virtudes e fraquezas dos recursos de conhecimento da organização em questão e as informações contidas neste produto servem de subsídio para o planejamento de ações relacionadas à Gestão do Conhecimento (FRESNEDA et al., 2008).

Para facilitar a compreensão e aplicação do método OKA, este foi dividido em: etapa inicial, etapa preparatória, etapa operacional e etapa final (figura 2).

Figura 2: Fases da aplicação do Método OKA

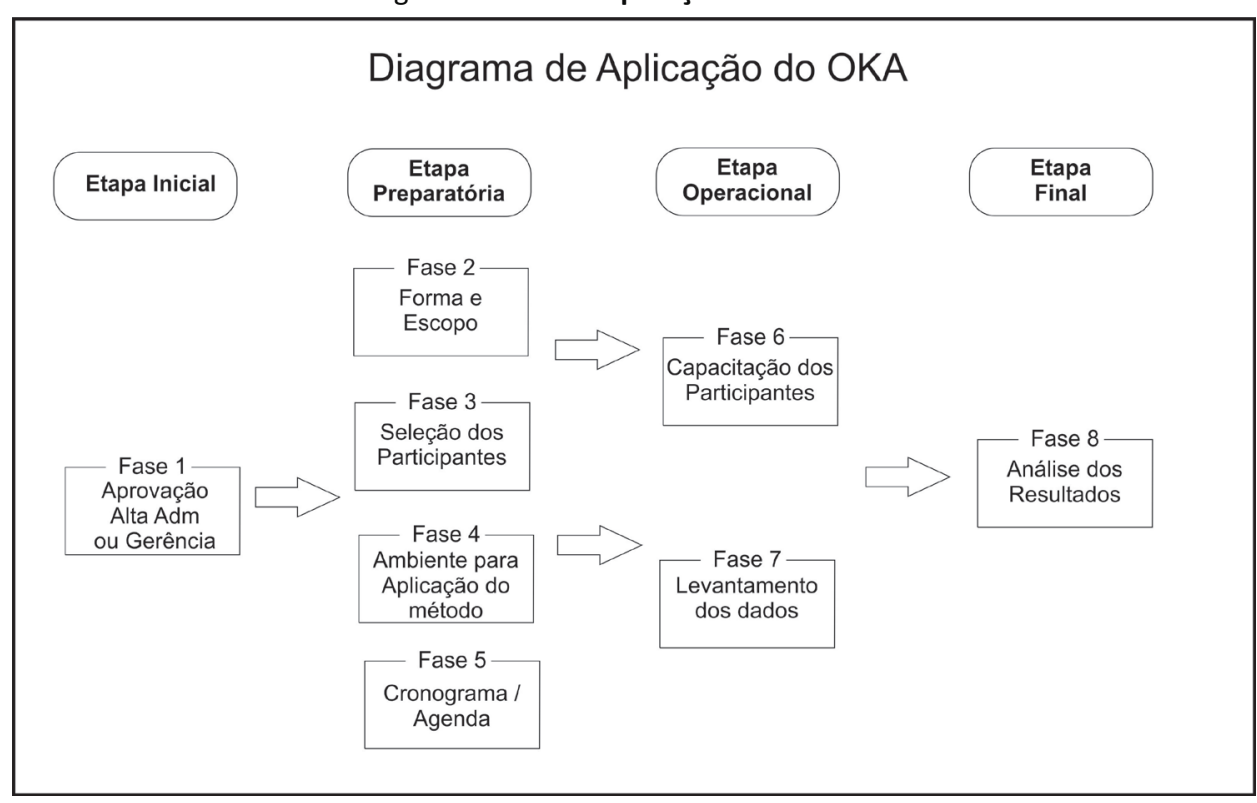

Fonte: Adaptado de Fonseca, 2006

É importante mencionar que no processo de resposta do questionário do método OKA será utilizada como forma de resposta a divisão por setores, permitindo a participação de vários colaboradores. A Defesa Civil de Santa Catarina possui os setores bem definidos, facilitando a aplicação do método e garantindo a qualidade das respostas de seus gestores. 


\section{ANÂLISE E RESULTADOS}

A Defesa Civil de Santa Catarina foi criada através da lei 4.841, de 18 de maio de 1973, vinculada ao Gabinete da Casa Civil, com afinidade direta ao Governador do Estado, na época Governador Colombo Machado Salles. O Estado de Santa Catarina, buscando assegurar o direito fundamental e constitucional do cidadão que é a segurança à vida, delega para a Defesa Civil o planejamento de ações que possam promover a defesa permanente contra as situações emergenciais (SECRETARIA DE DEFESA CIVIL, 2015).

A Secretaria de Estado da Defesa Civil foi criada pela Lei Complementar no 534, de 20 de abril de 2011. De Departamento Estadual de Defesa Civil, vinculado à Secretaria Executiva da Justiça e Cidadania e a Secretaria de Estado da Segurança Pública e Defesa do Cidadão, se transformou em Secretaria de Estado, em maio de 2011. Na estrutura da Secretaria de Estado de Defesa Civil, destacam-se a criação de duas Diretorias, sendo uma de Prevenção e outra de Resposta, para melhor realizar as ações de Defesa Civil e atender aos anseios da população. $O$ decreto no 553, de 27 de setembro de 2011, cria a Comissão Estadual de Prevenção, Preparação e Resposta Rápida a Emergências Ambientais com Produtos Perigosos (CE-P2R2), destinada a implantar e promover ações de prevenção, preparação e resposta rápida a acidentes ambientais com produtos perigosos (SECRETARIA DE DEFESA CIVIL, 2015).

A Defesa Civil catarinense busca aumentar a consciência e o compromisso em torno de ações que reduzam o risco de desastres. Dessa forma, através do Decreto Estadual no 728, de 13 de dezembro de 2011, cria 36 Coordenadorias Regionais de Defesa Civil, que irão funcionar junto à sede das Secretarias de Estado de Desenvolvimento Regional. No entanto, até a presente pesquisa, apenas 13 coordenadorias regionais foram ativadas no Estado.

Portanto, sua descentralização visa a redução dos desastres, o que compreende quatro ações distintas: ações de prevenção, ações de preparação para emergência, ações de resposta aos desastres e ações de recuperação aos eventos adversos que ocorre no território catarinense. Atua também de forma a incentivar a proatividade da sociedade para a redução de riscos de desastres em conjunto com as entidades e órgãos do município.

Sendo assim, nossa unidade de análise foi a Secretaria de Estado de Defesa Civil (SIEDEC), onde foi aplicado o método OKA com 199 questões com os diretores e gerentes da Secretaria, totalizando 6 funcionários e os dados foram tabulados da seguinte maneira: as questões que apresentaram, no mínimo, cinco alternativas como resposta foram logo consideradas como resposta de consenso; da mesma forma, para as que apresentaram convergência em quatro das seis. As questões restantes com diversidade de respostas, ou seja, não era maioria absoluta, foram levadas para discussão, onde os funcionários reunidos, chegaram ao consenso. Na sequência, as respostas foram lançadas no software de coleta de dados do método OKA.

\subsection{Diagnóstico Através do Método OKA}

O método de Avaliação da Gestão do Conhecimento Organizacional, desenvolvido pelo World Bank Institute (WBI, 2014), foi selecionado, a partir da apresentação de Fonseca (2006), como uma metodologia. O método OKA tem-se demonstrado de grande utilidade como instrumento de diagnóstico e de apoio na elaboração do plano de Gestão do Conhecimento, bem como, de forma indireta, acaba sendo um meio didático para a educação dos colaboradores em GC, informando-os sobre o que é GC e sua importância e benefícios às organizações públicas. $O$ método OKA ajuda a avaliar o estágio de desenvolvimento da GC nas organizações públicas. A 
estrutura básica do método, conforme esclarece o palestrante, é composta por três elementos: Pessoas, Processos e Sistemas. Estes elementos se compõem de dimensões do conhecimento e cada dimensão incorpora um conjunto de métricas, às quais, por sua vez, estão associadas perguntas de um questionário (FRESNEDA, 2006).

O método OKA, de acordo com Fresneda (2006), utiliza como métrica os seguintes fatores: o grau com que as políticas da organização recompensam as atividades ligadas ao conhecimento; a tolerância da organização com os riscos e comportamentos relacionados a ações de inovação; o grau com que a organização apoia e oferece atividades ligadas ao aprendizado de seus colaboradores; a receptividade da instituição a mudanças no trajeto profissional dos colaboradores; a participação dos colaboradores na melhoria do desempenho da organização; e a receptividade da empresa à ideias externas.

O Método OKA gera uma tabela e um gráfico do tipo radar. A primeira foi usada na análise dos elementos e suas dimensões e o segundo na conclusão da análise quantitativa. A tabela gerada pelo OKA possui duas colunas, onde na primeira coluna apresenta as 14 dimensões do conhecimento, e na coluna seguinte os valores disponibilizados pelo OKA, de maneira que se possa visualizar as diferenças de cada unidade pesquisada em relação ao grau de prática de GC para cada dimensão. Para facilitar a interpretação e comparação da tabela e do gráfico, usou-se o critério de Fonseca (2006, p.125), no qual os resultados foram transformados nos seguintes indicadores, quadro 1.

Quadro 1 - Indicadores das Métricas

\begin{tabular}{|ccc|}
\hline Índices & Conceito & Resultado \\
\hline $0-10$ & Baixo & Ponto fraco \\
\hline $11-20$ & Médio & Ponto neutro \\
\hline $21-30$ & Alto & \multirow{2}{*}{ Pontos fortes } \\
\hline Acima de 31 & Muito alto & \\
\hline
\end{tabular}

Fonte: Fonseca, 2006

É importante observar que as dimensões não necessariamente restringem-se a apenas um elemento. Ao contrário, podem impactar em dois deles ou até os três - Pessoas, Processos e Sistemas. Como exemplo, Fresneda et al. (2008) citam o compartilhamento de conhecimento, que aparece no elemento pessoas, mas contém certamente contribuições de tecnologias e políticas (processos). À tecnologia e as políticas do compartilhamento de conhecimento, neste caso, podem estar incluídas métricas específicas, tais como Infraestrutura de TI e Políticas de Qualidade em GC, respectivamente.

A interpretação do resultado da Secretaria de Estado de Defesa Civil, sua dimensão pelo método OKA e suas possíveis causas são descritas a seguir. 
Tabela 1 - Resultado da aplicação do método OKA

\begin{tabular}{c|c}
\hline \multicolumn{1}{c}{ SECRETARIA DE ESTADO DE DEFESA CIVIL DE SC } & Valor \\
\hline Dimensões & 19,4 \\
\hline Incentivos Culturais & 19,4 \\
\hline Criação e Identificação do Conhecimento & 12,6 \\
\hline Comunidades de Prática e Times do Conhecimento & 18,3 \\
\hline Conhecimento e Aprendizagem & 42,3 \\
\hline Liderança e Estratégia & 31,1 \\
\hline Fluxo do Conhecimento & 11,6 \\
\hline Operacionalização do Conhecimento & 23,8 \\
\hline Alinhamento & 20,3 \\
\hline Métricas e Monitoramento & 7,1 \\
\hline Tecnologia & 11,0 \\
\hline Infraestrutura de Acesso ao Conhecimento & 30,0 \\
\hline Conteúdo do Conhecimento & 26,8 \\
\hline Programa de Gestão do Conhecimento & 11,4 \\
\hline
\end{tabular}

Fonte: Elaborado pelos autores.

De acordo com Fonseca (2006) para efeito de análise, consideram-se como pontos fortes os valores superiores a 20 e como dimensões fracas os valores inferiores a 10 (quadro 1). Deste modo, foram observados os seguintes valores globais para a Secretaria de Defesa Civil:

Pontos fortes: Alinhamento (20,3); Operacionalização do Conhecimento $(23,8)$; Conteúdo do Conhecimento (26,8); Infraestrutura de Acesso ao Conhecimento $(30,0)$; Liderança e Estratégia $(31,1)$ e Conhecimento e Aprendizagem $(42,3)$.

Pontos Médios: Tecnologia (11,0); Programa de Gestão do Conhecimento (11,4); Fluxo do Conhecimento $(11,6)$; Compartilhamento do Conhecimento $(12,6)$; Comunidades de Prática $(18,3)$; Incentivos Culturais $(19,4)$ e Criação e Identificação do Conhecimento $(19,4)$.

Pontos Fracos: Métricas e Monitoramento $(7,1)$.

$\mathrm{Na}$ análise dos pontos fortes e fracos, partindo-se da imagem do diagrama final (gráfico 1), pode-se detalhar as informações geradas pelo método de avaliação Organizacional (OKA), começando pelas grandezas que estão relacionadas às dimensões indicadas e avançando sobre as respostas das questões componentes destas métricas. O diagnóstico obtido pelas práticas da Gestão do Conhecimento consolida num conjunto amplo de informações e possibilitará a elaboração de um Plano de Gestão do Conhecimento, alinhado à estratégia e aos objetivos da organização pública em questão. 
Instituição: Secretaria de Estado de Defesa Civil de Santa Catarina Data: 9 de janeiro de 2013

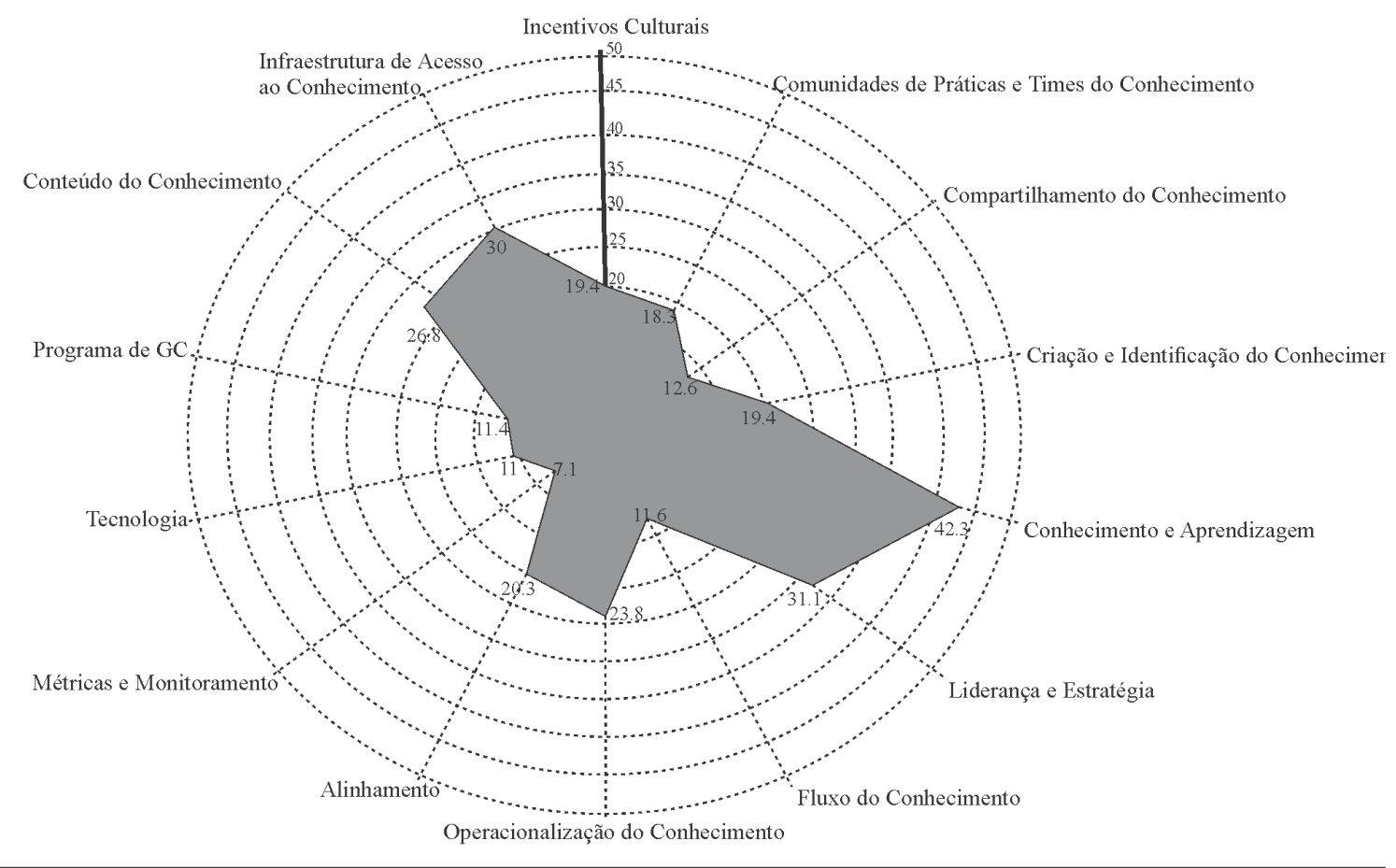

Fonte: Elaborado pelos autores.

\subsubsection{Elemento Pessoas}

Os Incentivos Culturais $(19,4)$ foram analisados nesta dimensão as atitudes culturais implícitas e explícitas, crenças e incentivos, que existem na organização para moldar, criar e apoiar o uso dos ativos intelectuais para alcançar as suas metas (FONSECA,2006). Foi observado um resultado médio, sendo este valor influenciado pela política de capacitação, realizada de forma individualizada, associada diretamente ao projeto em que o pesquisador está alocado. A cultura dos órgãos públicos necessita mudar para uma cultura de compartilhamento. Para que isso aconteça, faz-se necessário ser administrado por uma forte liderança, disponibilizando ferramentas de Gestão do Conhecimento e estimulando o uso do sistema e a compartilhar conhecimento, pois as pessoas precisam ser motivadas de forma adequada para contribuir.

Da mesma forma que a dimensão anterior, a Identificação e Criação do Conhecimento apresentam 19,4, mostrando a capacidade da organização e de seus controladores em identificar e criar entendimento e outros ativos intelectuais (PARK; KIM, 2005). Este espaço não necessariamente precisa ser físico, pois as interações entre as pessoas podem acontecer por meios virtuais, com fins de incentivar o compartilhamento das informações, a criação do saber e o trabalho em equipe. No entanto, o Compartilhamento do Conhecimento $(12,6)$ mostra a baixa capacidade da organização e de seus controladores para compartilhar ativos intelectuais que a permitem atingir seus objetivos (FONSECA, 2006). Esta avaliação ocorre em função da estrutura extremamente funcional, hierárquica e da falta de prioridade à construção de redes sociais para o compartilhamento do conhecimento. 
Já as Comunidades de Prática e Equipes de Conhecimento $(18,3)$ ressaltam a existência, natureza e utilização de grupos de pessoas dentro da empresa que possam, efetivamente, alavancar a solução de problemas (ROSSETTI, MORALLES, 2012). Foram apresentados índices razoáveis, pela falta de incentivo à criação de equipes do conhecimento e comunidades de prática, bem como a promoção para criação de ambientes colaborativos.

De forma contrária, as dimensões Conhecimento e Aprendizado $(42,3)$ que refletem a existência de treinamento e outras atividades de conhecimento, dirigido formalmente ou estruturado na organização e a sua capacidade na formação do capital humano (FONSECA, 2006). Os valores elevados demonstram o quanto a Secretaria de Defesa Civil está preocupada em dar condições de capacitação aos seus funcionários, para que estes tenham potencial para atingir os objetivos da organização. Nesta dimensão, ocorre grande investimento por parte do governo para qualificar melhor seus colaboradores da Secretaria de Estado de Defesa Civil, com programas de cooperação com outros Estados e parceria com a União, como forma de aprendizagem pelas experiências vividas em situações adversas.

Para Garvin (2003), as organizações que aprendem com seus ativos intelectuais, devem estar capacitadas a criar, adquirir e transferir conhecimentos e em modificar comportamentos para refletir estes novos saberes. Desta forma, a Secretaria de Defesa Civil de Santa Catarina, por ser uma organização de articulação e desenvolvimento, privilegia muito a capacitação de seus funcionários, demonstrada na dimensão Conhecimento e Aprendizagem, na qual são verificadas a existência e a capacidade da organização para a edificação do capital humano através de treinamento ou desenvolvimento formalizado na construção do conhecimento. Contudo, ao observar o índice de 12,6 apresentado no gráfico 1, referente à dimensão Compartilhamento do Conhecimento, verifica-se a necessidade de criar programas e estímulos para que seus ativos intelectuais possam ser compartilhados com os demais interessados, principalmente fora de seus setores, sendo necessário o diálogo com outras áreas da Secretaria de Defesa Civil. Esta prática irá aproximar os colaboradores e resultará na melhor qualidade do serviço prestado.

Analisando todas as dimensões dos elementos Pessoas, observa-se que todos os resultados estão classificados como de médio a elevado, com uma média na dimensão Pessoas de 22,4. Dessa forma, e de acordo com o método OKA, pode-se dizer que a empresa diagnosticada está num nível forte de identificação, compartilhamento e aprendizado em relação à Gestão do Conhecimento (FONSECA, 2006).

\subsubsection{Elemento Processos}

Para o elemento Processo, interpretou-se que Liderança e Estratégia (31,1), onde representa a adoção e execução da Gestão do Conhecimento como um princípio gerencial pela liderança de seus colaboradores, destacando-se como um valor corporativo, e permitindo estar apta e flexível a mudanças em sua própria estrutura, retratando num bom relacionamento entre a alta direção e o corpo gerencial da organização (FONSECA, 2006). Neste aspecto, pode-se observar que a dimensão Liderança e Estratégia exprime a imagem da Secretaria de Estado de Defesa Civil. Esta constatação é evidenciada, pois a Secretaria possui uma estrutura altamente hierarquizada, em sua maioria funcionários de origem de organizações militar, como Polícia Militar e Corpo de Bombeiros Militar, nos quais exercem funções na parte operacional e gerencial.

As dimensões Fluxo do Conhecimento $(11,6)$ concernem ao conjunto completo de informações, dados e relações que levam as pessoas à tomada de decisão, à realização de tarefas e à criação de novas informações ou conhecimentos (FIALHO, 2006). Apesar de o índice correspon- 
der como ponto médio, esse sem dúvida é um dos grandes desafios da Defesa Civil no Estado de Santa Catarina: a capacidade da organização fazer fluir o conhecimento e outros ativos intelectuais (incluindo captura, armazenamento e disseminação) como forma de chegar aos municípios e a partir destes serem usados na prevenção e resposta aos desastres. Esta integração de forças corporativas irá ocorrer se houver o agrupamento das práticas da Gestão do Conhecimento nas categorias, Pessoas, Processos e Sistemas, permitindo que pessoas ajudem a operacionalizar recursos com o uso das tecnologias, enquanto processos definem as funções e necessidades e instigam o conhecimento necessário nas pessoas (EDWARDS, 2009).

A Operacionalização do Conhecimento $(23,8)$ na qual é analisar a capacidade da organização em integrar e aplicar conhecimento nos seus negócios e processos operacionais, tais como o grau com que os processos da organização estão documentados e acessíveis aos empregados (FONSECA, 2006). Pelos valores obtidos, pode-se afirmar que as informações dentro de cada divisão de projeto se encontram disponíveis, mas o compartilhamento do conhecimento na organização não é praticado. Deste modo, revela a necessidade de uma mudança significativa na transformação das práticas de conhecimento junto aos seus negócios e processos operacionais, pois não existe uma integração desses processos e conhecimentos em toda a empresa, é o que revela a Operacionalização do Conhecimento $(23,8)$.

Já o Alinhamento $(20,3)$, que mede o grau de conhecimento dos objetivos e resultados do programa de Gestão do Conhecimento com os objetivos da empresa (FONSECA, 2006), mostra um valor alto. Isso ocorre mesmo a secretaria não possuindo um programa formal de Gestão do Conhecimento. Entretanto, devido sua atividade específica, ocorre algumas ações planejadas e executadas de forma articulada, isolada e sinérgica.

A dimensão Métricas e Monitoramento $(7,1)$, que identifica a capacidade da organização em gerenciar os ativos intelectuais, monitorar e identificar as melhores práticas, informações externas e aprendizado (FONSECA, 2006), mostra o grande fator contraproducente e que necessita de maior atenção por parte da Secretaria. Em decorrência da natureza e composição da Secretaria de Estado de Defesa Civil, não existem indicadores estabelecidos para mensurar resultados em investimentos no conhecimento.

Analisando todas as dimensões do elemento Processo, observa-se que a organização alvo operacionaliza o conhecimento relativamente bem, porém as demais dimensões desse elemento, as quais representam grande parte da Gestão do Conhecimento, apresentam valores baixos, como captura, armazenamento e disseminação do conhecimento.

\subsubsection{Elemento Sistemas}

No elemento Sistema, no concernente a Dimensão Tecnologia da GC $(11,4)$, é analisado a existência e a capacidade de infraestrutura tecnológica que permitam o compartilhamento de melhores práticas dentro da GC. Estão incluídos nesta dimensão os softwares, aplicações ou ambientes de comunicação existentes; a telefonia e a infraestrutura tecnológica (FONSECA, 2006). Verifica-se o uso de videoconferência e Rede Internet. A prática da primeira, ela ocorre circunstancialmente, no entanto há uma carência de uma rede de dados de alta velocidade. Atualmente é utilizada a estrutura da UFSC, através do CEPED.

Sobre a infraestrutura organizacional, Davenport e Prusak (2003) citam a importância de ter um conjunto de funções, estruturas organizacionais e qualificações que beneficiem cada projeto. Para isso, o Governo Estadual vem implantando na Secretaria e Regionais internet de alta velocidade, aumentando sua banda larga e contribuindo para melhorar os processos administrativos, 
bem como facilitando a capacitação através do Ensino a Distância. Os dados apresentados pelo método OKA corroboram com essa deficiência, apresentando como índice 11 pontos, representando um ponto fraco no elemento Sistemas e necessita que seja revista toda a sua estrutura de TI.

Outro índice que se mostrou bastante baixo está na Infraestrutura do Ambiente de GC, com 11,4 pontos. Mesmo estando ambos classificados como médio, mostram sua vulnerabilidade e deficiência, estando muito próximos a serem considerados como ponto fraco. A flexibilidade na estrutura de TI de empresas públicas está positivamente associada a maior geração e disseminação de informação, que por sua vez, influenciam a capacidade da organização responder novas demandas e desafios (BHATT; EMDAD; ROBERTS; GROVER; 2010).

Contudo, é importante destacar na Dimensão Sistemas, a Infraestrutura de Acesso ao Conhecimento (30), na qual são analisadas a capacidade e infraestrutura existentes que permitem aos controladores acessarem e interagirem com os ativos intelectuais da organização (SILVA; MUSSI, 2014). Como forma de compreender este bom desempenho apresentado no método OKA, percebe-se que a Secretaria possui um ambiente de software adequado às suas necessidades organizacionais, tais como softwares de buscas institucionais e aplicativos de uso diário na administração. No entanto, verificou-se que a Defesa Civil possui poucos processos administrativos que contemplam os registros ou arquivos visando criar uma memória organizacional. Notadamente, não ocorrem incentivos para o compartilhamento do conhecimento entre os funcionários, apenas poucas práticas informais, que não são percebidas por seus colaboradores. Isso é corroborado com a Dimensão Gestão do Conteúdo com 26,8, que apresenta valores significativos que mostram a criação de conteúdo dentro da organização.

Especialmente com ativos intelectuais, que são pouco explorados, compartilhados e, muitas vezes, com vários colaboradores sem saber da existência desses ativos e sem se preocupar com a forma de registrar o conhecimento ou a informação.

A dimensão Conteúdo do Conhecimento $(26,8)$, em que são analisados os tipos de conteúdo e as ferramentas de gestão da informação que a organização produz ou gerencia para executar a gestão do conhecimento (FONSECA, 2006), apresentou uma pontuação muito alta em relação às outras dimensões. No entanto, como a organização alvo não possui um programa formal de GC, ela não tem uma infraestrutura tecnológica adequada à Gestão do Conhecimento.

Já na dimensão de Programa de Gestão do Conhecimento $(11,4)$, na qual são analisados a natureza, o desenho e a capacidade do programa de Gestão do Conhecimento, envolvendo pessoas, unidades e grupos na organização (FONSECA, 2006), foi obtido um fator total de 11,4, o que configura um resultado médio. Apesar da instituição possuir alguns mecanismos funcionais de gestão do conhecimento, como a gestão eletrônica de documentos, recém-implantada; algumas informações internas disponibilizadas em rede e um programa de acesso a normas de elaboração de requisitos técnicos para projetos, pode ser afirmado que a empresa não possui um programa formal de Gestão do Conhecimento.

\section{CONSIDERAÇÕES FINAIS}

A Gestão do Conhecimento em uma organização deve ser fruto de uma gestão estratégica, levando em consideração todos os seus aspectos, para que se tenha o melhor resultado possível e possa alcançar todos os benefícios que ela pode proporcionar. As iniciativas de Gestão do Conhecimento têm o potencial de auxiliar as organizações a identificar, adquirir, desenvolver, compartilhar e usar o conhecimento e a expertise dos seus colaboradores e o gerado dentro e fora da organização.

Dessa forma, a Gestão do Conhecimento no setor público também é muito importante, 
com o objetivo do melhor funcionamento da máquina pública e a eficiência dos serviços prestados aos cidadãos. Para que o conhecimento seja um recurso básico na busca da efetividade das ações públicas no Brasil, faz-se necessária a formulação de políticas de conhecimento que ajudem os órgãos e empresas públicas a realizarem suas estratégias.

Este artigo contribuiu para compreender melhor as práticas da Gestão do Conhecimento na Secretaria de Defesa Civil de SC, tendo como fator positivo a dimensão do Conhecimento e Aprendizagem, na qual se destacou a capacidade da organização para a construção do capital humano, utilizando diversos programas de capacitação, principalmente após a parceria com o Centro Universitário de Estudos e Pesquisas sobre Desastres - CEPED, onde foi possível o desenvolvimento de programas de mapeamento de áreas de risco e a capacitação de colaboradores ao longo do Estado, abrangendo todos os municípios.

Outro ponto a ser destacado de forma positiva encontra-se na dimensão Liderança e Estratégia, pois sua estrutura é altamente hierarquizada, onde a maioria dos funcionários é de origem de organizações militares, como Polícia Militar e Corpo de Bombeiros, instituições que tem como princípios basilares a hierarquia e a disciplina. Deste modo, verificou-se que alguns cargos estratégicos são ocupados considerando seu posto na hierarquia militar. Isso, de certa forma, pode levar a antiguidade a sobrepujar os valores de competência, criando um desestímulo nos demais colaboradores que não estão no contexto de origem dos órgãos militares.

Outra constatação que convém mencionar está no elemento pessoas, o qual necessita ser trabalhado nas comunidades de práticas em função da diversidade cultural, sendo necessária uma forte liderança com uso de ferramentas apropriadas, para que possa estar à frente do programa, estimulando e orientando os colaboradores a contribuir com o compartilhamento do conhecimento na Secretaria de Estado de Defesa Civil.

No caso estudado, constata-se que a cultura organizacional e a falta de conhecimento mais profundo em relação às técnicas de GC são fatores que impactam com maior intensidade na formalização de práticas de Gestão do Conhecimento.

Dentre alguns obstáculos que devem ser vencidos, para que o programa de GC possa ser implantado na Defesa Civil de Santa Catarina, podem ser citados: Reconhecimento da alta direção da importância da GC na área de Defesa Civil; melhoramento da infraestrutura de Tecnologia da Informação e Comunicação, além de Sistemas específicos para gestão em toda a Secretaria; falta de um programa de capacitação em GC aos colaboradores; falta de compreensão sobre Gestão do Conhecimento de seus diretores e gerentes; falhas nas ferramentas de comunicação; atitudes isoladas em detrimento ao grupo e falta de incentivos para compartilhar conhecimento.

Portanto, através deste estudo na Secretaria de Estado de Defesa Civil de Santa Catarina, foi possível fazer um diagnóstico nas práticas de Gestão do Conhecimento e, desta forma, elaborar a partir dos dados obtidos um Programa de Gestão do Conhecimento. No entanto, é importante destacar que diversas práticas vêm sendo executadas nos setores, com o grande desafio de transferir da informalidade para um processo diário de compartilhamento do conhecimento no âmbito Estadual, permitindo melhores respostas as ações de desastres e contribuindo no restabelecimento da normalidade social.

Apesar da importância dos resultados obtidos, deve ser mencionado e reconsiderado para outras investigações a aplicação do método OKA, pois mesmo abrangendo todos os setores e seus gerentes, pode ser aplicado aos demais funcionários e, desta forma, verificar os resultados entre os diferentes níveis funcionais da Secretaria de Defesa Civil. Assim, ressalta-se que a principal 
contribuição desta pesquisa consiste em demonstrar como ocorrem as práticas de GC que, em meio a um conjunto de interações, mesmo que informalmente, mostram as deficiências e os valores organizacionais, e apresenta-se como imprescindível para o processo de construção da GC.

Sob a ótica acadêmica, fica como desafio para trabalhos futuros a continuação deste projeto, a partir dos resultados obtidos para a implantação de um Programa de Gestão do Conhecimento, bem como a adoção de mecanismos para mensurar o desempenho da GC na Defesa Civil catarinense, aprofundando os estudos em todas as regiões do Estado.

\section{REFERENCIAS}

ANSUATTIGUI, R. V; PITHON, A. J. C. Aplicação do Método OKA em Redes Colaborativas de Autoria. In: XXXI ENCONTRO NACIONAL DE ENGENHARIA DE PRODUÇÃO (ENEGEP), p. 1-12, Belo Horizonte, 2011.

BATISTA, F. F; QUANDT, C. O; PACHECO, F. F; TERRA, J. C. C. Gestão do Conhecimento na Administração Pública. Texto para discussão n. 1095. Brasília: IPEA, 2005.

BHATT, G. D. Knowledge Management in organizations: examining the interaction between, technologies, techniques, and people. Journal of Knowledge Management, v. 5, n. 1, p. 68-75, 2001.

BHATT, G. D; EMDAD, A; ROBERTS. N. \& GROVER, V. Building and lever-aging information in dynamic environments. Information \& Management, 47(7-8), p. 341-349, 2010.

BIERLY, P, CHAKRABARTI, A. Generic knowledge strategies in the US pharmaceutical industry. Strategic Management Journal, p. 123-135, 1996.

BIERLY, P. \& DALY, P. Aligning human resource management practices and knowledge strategies: Atheoretical framework. In: C.W. Choo \& N. Bontis (Eds.), The strategic management of intellectual capital and organizational knowledge. Oxford University Press, 2002.

BOFF, L. H. Conhecimento: Fonte de riqueza pessoas e organizações. Fascículo profissionalização, v. $22,2000$.

BUKOWITZ, W. R; WILLIAMS, Ruth L. Manual de Gestão do Conhecimento: ferramentas e técnicas que criam valor para a empresa. São Paulo: Bookman, 2002.

CALHEIROS, L. B; CASTRO, A. L. C. e DANTAS, M. C. A Implantação e Operacionalização de Comdec. Ministério da Integração Nacional, Secretaria Nacional de Defesa Civil. Brasília, 2009.

CRUZ, T. Gerência do conhecimento. São Paulo: Cobra, 2002.

DAVENPORT, T. H; PRUSAK, L. Conhecimento empresarial: como as organizações gerenciam o seu capital intelectual. Rio de Janeiro: Elsevier, 2003.

DAVENPORT, T. H; VÖLPEL, S. The rise of knowledge towards attention management. Journal of Knowledge Management, 5(3), 212-221, 2001.

EDWARDS, J. S. Business processes and Knowledge management. In M. Khosrow-Pour, 
Encyclopedia of Information Science and Technology, 2 ed. v. 1, Hershey, PA: Igi Global, 2009.

FIALHO, F. A. P; MACEDO, M; SANTOS, N; MATIDIERI, T.C. Gestão do Conhecimento e aprendizagem: as estratégias competitivas da sociedade pós-industrial. Florianópolis: Visual Books, 2006.

FONSECA, A. F. Organizational Knowledge Assessment Methodology. Washington, DC: World Bank Institute, 2006.

FONSECA, A. F; TORRES, M. F. P; GARCIA, J. C. R. Definição de Referências e Adequação do Uso do Método OKA na Medição dos Elementos Necessários para Gestão do Conhecimento em Organizações de Pequeno e Médio Porte. II Simposio Iberoamericano em Generación, Comunicación y Gerencia del Conocimiento: GCGC 2010.

FRESNEDA, P. S.V; GONÇALVES, S. M. G. A experiência brasileira na formulação de uma proposta de política de Gestão do Conhecimento para a Administração Pública Federal. Brasília. Câmara dos Deputados, Coordenação de Publicações, 2007.

FRESNEDA, P. S. V; GONÇALVES, S. M. G; PAPA, M; FONSECA A. F. Diagnóstico da Gestão do Conhecimento nas Organizações Públicas Utilizando o Método Organizational Knowledge Assessment (Oka). In: Il Congresso Consad de Gestão Pública-Painel 20: Gestão do Conhecimento e inovação para a melhoria da gestão pública, 2008.

GARAVELLI, C; GORGOGLIONE, M; SCOZZI, B. Knowledge management strategy and organization: A perspective of analysis. Knowledge and Process Management, p. 273-282, 2004.

GARVIN, D. A. Building a learning organization. Harvard Business Review, 71(4), jul-aug. 2003.

GROTTO, D. Um olhar sobre a Gestão do Conhecimento. Revista de Ciências da Administração. Florianópolis, ano 3, n. 6, p. 31-37, 2001.

LEOCÁDIO, L. C. S. Mecanismos de Coordenação e práticas da Gestão do Conhecimento na Rede de Valor Terceirizada: Estudo no Setor Elétrico, 2011. 169 p. Tese Departamento de Engenharia e Gestão do Conhecimento, UFSC, Florianópolis, 2011.

$\mathrm{MARCH}$, J. G. Exploration and exploitation in organizational learning. Organization Science, 2(1), 71-87, 1991.

MARCONI, M. A; LAKATOS, E. M. Fundamentos de metodologia científica. 6a ed. São Paulo: Atlas 2005.

NONAKA, I; TAKEUCHI, H. The Knowledge-Creating Company: How Japanese Companies Create the Dynamics of Innovation (Hardcover). New York: Oxford University Press, 1995.

Criação de conhecimento na empresa: como as empresas japonesas geram a dinâmica da inovação. Rio de Janeiro: Campus, 1997.

PARK, Y; KIM, S. Linkage between knowledge and R\&D management. Journal of Knowledge Management, v. 9, n. 4, 2005.

PEREZ-SOlTERO, A, VALENZUELA, M.B. SANCHEZ-SCHMitZ, F.M.R, PALMA-MENDEZ, J. T. \& VANTI, A. A, Knowledge Audit Methodology with Emphasis on Core Processes. European and Mediterranean Conference on Information System (EMCIS), Costa Blanca, Alicante, Spain, 6-7 
July, 2009.

PINSKY, V. C.; KRUGLIANSKAS, I. Gestão estratégica da sustentabilidade e inovação. Rev. Adm. UFSM, Santa Maria, v. 6, número 3, p. 465-480, set. 2013.

ROSSETTI, A, \& MORALES, A. B. Sistemas de computação do usuário final: apoio direto à computação para produtividade do usuário final e colaboração de grupos de críticas para a alta gerência. Sistemas especialistas: conselho especializado baseado em conhecimento para os usuários. Knowledge Management, p. 1-10, 2012.

SANTOS, I. C; AMATO NETO, J. Gestão do Conhecimento em Indústria de Alta Tecnologia. v. 18, n. 3, p. 1-13, 2012.

SCHREIBER, G. et al.. Knowledge engineering and management: the commonKADS methodology. MIT Press, 2002.

SCHULTZ, M. \& Jobe, L. A, Codification and tacitness of knowledge management strategies: An empirical exploration, Journal of High Technology Management Research 12(1), p. 139-166, 2011.

SECRETARIA DE DEFESA CIVIL. A Estrutura da Secretaria de Defesa Civil em SC. Disponível em: http://www.defesacivil.sc.gov.br/. Acesso em: 27 jun 2015.

SILVA, I; MUSSI, C. Tecnologia da Informação, Criação e Compartilhamento do Conhecimento: Um Estudo do Sistema Integrado de Segurança Pública em Santa Catarina. Rev. Adm. UFSM, Santa Maria, v. 7, número 1, p. 81-100, mar. 2014.

SILVEIRA, R. R. Diretrizes para mitigar as Barreiras à Implementação da Gestão do Conhecimento em organizações. 2011, p. 221, Tese Engenharia e Gestão do Conhecimento, UFSC, Florianópolis, 2011.

WIIG, K. M. Introducing Knowledge management into the enterprise. In: LIEBOWITZ, Jaz. Knowledge management handbook. USA, CRC press LLC, 2000.

WORLD BANK INSTITUTE. About the World Bank Institute. Disponível em: http://go.worldbank. org/53LOBQ2OKO. Acesso em: 20 abril 2014.

ZACK, M. H. Developing a knowledge strategy. California Management Review, v. 41, n. 3, p. 125 , 1999. 